Kalpa Publications in Engineering
Volume 3, 2020, Pages 139-149
Proceedings of International Sym-
posium on Applied Science 2019

\title{
Bending analysis of sandwich beam with functionally graded face sheets using various beam theories by meshfree method
}

\author{
Minh Duc Do ${ }^{1}$, Minh Tu Tran ${ }^{2 *}$, Hoai Chinh Truong ${ }^{1}$ \\ ${ }^{1}$ Faculty of Civil Engineering, The University of Da Nang, Da Nang, Viet Nam \\ ${ }^{2}$ National University of Civil Engineering, Ha Noi, Viet Nam \\ tutmanuce.edu.vn
}

\begin{abstract}
An interpolation technique-based meshfree method using polynomial functions is employed to analyze static behavior of sandwich beams with functionally graded face sheets and homogenous soft core. Various beam theories are expressed in general form and taken into account both shear deformation and normal deformation effects. Governing equation is derived from the principle of virtual work. The obtained results have been verified with the previously published works and a good agreement is found. The effects of skin-core-skin thickness ratios, material volume fraction indices, slenderness ratios, shear deformation and thickness stretching effect on deflection and axial stress are investigated and discussed.
\end{abstract}

\section{Introduction}

Due to high stiffness-to-weight ratio, high strength-to-weight ratio as well as possessing good properties such as resistance to corrosion, abrasion or heat transfer, sound and energy absorption, etc., sandwich structures have been widely applied in many areas of aerospace, space, construction, machine elements, etc. [1]

A typical sandwich structure usually consists of two skin layers and a core embedded between them that deliberately promotes good material properties of each layer. Commonly, material of each layer is homogeneous, isotropic but completely different from layer to layer which insults in sudden changes in the properties at the contact face between layers. This causes stress concentration and leads to failure or separation. Those drawbacks could be avoided by using materials in which their mechanical properties change gradually. Functionally graded materials (FGMs) [2, 3] recently have developed as the potential candidates satisfying the above requirements. Sandwich structures made of

${ }^{*}$ Corresponding author.

T.T. Truong, T.N. Tran, Q.K. Le and T.N. Nguyen (eds.), ISAS 2019 (Kalpa Publications in Engineering, vol. 3), pp. 139-149 
FGMs are named FG sandwich structures. So far, FG sandwich structures such as beams, plates have been used in many high-tech fields $[2,3]$ and promises great potential application in the near future.

Simulation for prediction of the mechanical response is an important part to aid the experiment, design, fabrication or operation of the structures. Moreover, for structures with novel materials, this topic also attracts research efforts to reveal and implement strange behaviors. To this end, it is possible to use several computational methods which could be divided into two major groups. The first group is analytical solutions, which gives ideal results but has challenges with complex issues. Numerical methods, belonging to the second group with approximation solution, allow to overcome the problems of analytical solutions. Many numerical methods which have been developed and proposed for these purposes such as Ritz method, finite element method (FEM), meshfree method, shooting method, Lagrange multiplier method, state space method, etc., was reviewed in great detail by Sayyadandy and Ghugal [2]

Meshfree method has been developed and applied over three decades. It is proved to be an effective numerical solution, especially for complex structural problems that FEM has challenges [4]. There have been several different structural problems successfully simulated employing this method. However, for a class of complicated engineering problems about mechanical properties as FG sandwich beams, using this method is very limited, only a few reports are found: Karamanli [5] employed a kind of mesh free methods, namely symmetric smoothed particle hydrodynamics method, to analyze elastostatic behavior of the two directional FG sandwich beams based on a quasi-3D theory. Amirani et al. [6] used the element free Galerkin method and Galerkin formulation for twodimensional elasticity problems to investigate free vibration of sandwich beams; Bui et al. [7] proposed a truly meshfree method for dynamic analysis of sandwich beams with functionally graded core and homogeneous face sheets. The displacement field is approximated via radial basis function without predefined mesh, and the domain integrals are determined by the Cartesian transformation method to obviate the need for a background cell; Yang et al. [8] applied a meshfree boundarydomain integral equation method to investigate free vibration behavior of FG sandwich beams. Radial integration method was used to transform the domain integrals related to material non-homogeneity and inertia effect into boundary integrals.

Euler-Bernoulli beam theory (CBT) is simple, but only suitable for thin beams. For thick beams, neglecting shear deformation does not fully reflect the actual behavior and lead to overestimate loadcarrying capacity of beams. Therefore, several different beam theories have been proposed to take into account the effects of this deformation: first-order beam theory (FBT) - also known as Timoshenko beam theory, high-order beam theories (HBTs), and most recently, quasi-3D theory with combining both shear deformation and thickness stretching effects $[5,9,10,11,12]$. Overview of these theories and their mathematical formulations are presented in detail in Ref. [2].

In this paper, static behavior of FG sandwich beams is simulated by a meshfree method using point interpolation technique and polynomial basic function. The beams consist of a homogeneous soft core and two FGM face sheets whose mechanical properties vary continuously according to the power law in the depth-direction. Different beam theories expressed in general form are taken into account both shear deformation and normal deformation effects. The equilibrium equation is derived from the principle of virtual work. Polynomial basic function is employed to construct shape functions and approximate the global displacement field of computational domain. Numerical examples are conducted to verify the accuracy and evaluate the effectiveness of the computational method. Deflections, stresses are also investigated in detail with consideration the effects of skincore-skin thickness ratios, material volume fraction indices and beam theories. 
Bending Analysis of Sandwich Beam with Functionally Graded Face Sheets Using ... M. D. Do et al.

\section{Mathematical formulations}

\subsection{FG sandwich beam}

Consider an FG sandwich beam of dimensions $L \times b \times h$ having two face sheet layers composed of FG material and a full metal core layer - homogenous soft-core type, as shown in Figure 1. The layers are represented by numeric indicators 1,2 , and 3 . The top and bottom surfaces are located at $z= \pm h / 2$, respectively. The beam is named according to the thickness ratio among the skin-coreskin; for example, 1-2-1 beam is the sandwich beam whose core thickness doubles the face sheet thickness.

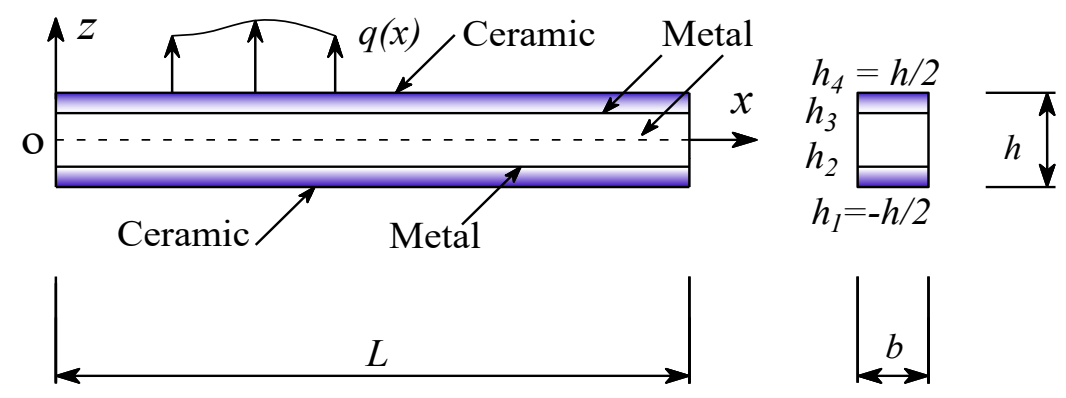

Figure 1: FG sandwich beam with geometric parameters and coordinate

Assuming Poisson's ratio $v$ is constant while Young's modulus $E$ is changed in each layer of the beam along the $z$-axis according to the power-law distribution:

$$
\begin{array}{ll}
E^{(1)}(z)=\left(E_{m}-E_{c}\right)\left(\frac{h_{4}-z}{h_{4}-h_{3}}\right)^{k}+E_{c}, & z \in\left[h_{3}, h_{4}\right] \\
E^{(2)}(z)=E_{m}, & z \in\left[h_{2}, h_{3}\right] \\
E^{(3)}(z)=\left(E_{m}-E_{c}\right)\left(\frac{z-h_{1}}{h_{2}-h_{1}}\right)^{k}+E_{c}, & z \in\left[h_{1}, h_{2}\right]
\end{array}
$$

where the superscript (i) denotes the $i$-th layer; $E_{c}$ and $E_{m}$ are Young's modulus of ceramic and metal constituents of FG materials, respectively; $k$ is the volume fraction index, $k \geq 0$.

\subsection{Displacement, deformation and stress fields}

The general displacement field, which includes transverse shear deformation and normal deformation effects according to quasi-3D beam theory, could be described in Eqs. (2)-(3) below

$$
\begin{aligned}
& \{d\}=\left\{\begin{array}{l}
u \\
w
\end{array}\right\}=\left[\begin{array}{ccccc}
1 & 0 & -z & \Phi(z) & 0 \\
0 & 1 & 0 & 0 & \Phi_{, z}(z)
\end{array}\right]\left\{A_{1}\right\}, \\
& \left\{A_{1}\right\}^{T}=\left\{u_{o}(x) \quad w_{o}(x) w_{o, x}(x) \phi_{o s}(x) w_{o z}(x)\right\},
\end{aligned}
$$

in which $u_{o}, w_{o}, w_{o z}$ are the displacements of a point at the mid-plane $(z=0) ; \phi_{o s}$ is the shear strain of the beam at the mid-plane; $\Phi(z)$ is the shape function which describes the distribution of the shear 
Bending Analysis of Sandwich Beam with Functionally Graded Face Sheets Using ... M. D. Do et al.

strain through the beam depth; $(\bullet)_{, x}$ and $(\bullet)_{, z}$ stand for the derivatives $\partial(\bullet) / \partial x, \partial(\bullet) / \partial z$; the exponent $(\bullet)^{T}$ represents the transpose.

By setting $w_{o z}(x)=0$ and choosing appropriate functions $\Phi(z)$, other different beam theories could be received as: $\Phi(z)=0$ for classical beam theory (CBT), $\Phi(z)=z$ for first-order beam theory (FBT), $\Phi(z)=z-4 z^{3} / 3 h^{2}$ for third-order beam theory (TBT).

The strain field associated with the displacement field in Eq. (2) are expressed as

$$
\begin{aligned}
& \{\varepsilon\}=\left\{\begin{array}{c}
\varepsilon_{x} \\
\varepsilon_{z} \\
\gamma_{x z}
\end{array}\right\}=\left[\begin{array}{cccccc}
1 & -z & \Phi(z) & 0 & 0 & 0 \\
0 & 0 & 0 & \Phi_{, z z}(z) & 0 & 0 \\
0 & 0 & 0 & 0 & \Phi_{, z}(z) & \Phi_{, z}(z)
\end{array}\right]\left\{A_{2}\right\}, \\
& \left\{A_{2}\right\}^{T}=\left\{u_{o, x}(x) \quad w_{o, x x}(x) \quad \phi_{o s, x}(x) w_{o z}(x) \phi_{o s}(x) \quad w_{o z, x}(x)\right\}
\end{aligned}
$$

The stress field could be obtained from Hooke's law and is expressed in matrix form below

$$
\begin{gathered}
\{\sigma\}=\left\{\begin{array}{l}
\sigma_{x} \\
\sigma_{z} \\
\tau_{x z}
\end{array}\right\}=\left[\begin{array}{ccc}
Q_{11} & Q_{12} & 0 \\
Q_{21} & Q_{22} & 0 \\
0 & 0 & Q_{33}
\end{array}\right]\left\{\begin{array}{c}
\varepsilon_{x} \\
\varepsilon_{z} \\
\gamma_{x z}
\end{array}\right\}=\left[E_{d}\right]\{\varepsilon\}, \\
Q_{11}=Q_{22}=\frac{E(z)}{\left(1-v^{2}\right)} ; Q_{12}=Q_{21}=Q_{11} v ; Q_{33}=G(z)=\frac{E(z)}{2(1+v)}
\end{gathered}
$$

\subsection{Energy expressions of the beam}

The virtual work done by internal force on virtual strain:

$$
\delta \mathrm{W}^{\text {int }}=\int_{V}\{\sigma\}^{T}\{\delta \varepsilon\} . d V
$$

Applying Hooke's law (Eq. (6)) and substituting Eq. (4) into Eq. (8), lead to:

$$
\delta \mathrm{W}^{\mathrm{int}}=\int_{V}\{\varepsilon\}^{T}\left[E_{d}\right]\{\delta \varepsilon\} . d V=\int_{0}^{L}\left\{A_{2}\right\}^{T}[D]_{E}\left\{\delta A_{2}\right\} d x,
$$

$[D]_{\mathrm{E}}$ is the matrix of dimension $(6 \times 6)$ containing elastic coefficients which are determined through $E(z)$ and $v ; V$ is the volume of the beam.

The virtual work done by external distributed load $q(x)$ on virtual displacement:

$$
\delta W^{e x t}=\int_{0}^{L} q(x) \cdot \delta w_{o}(x) d x
$$

\subsection{Approximation of general displacement field in the beam}

In this section, a meshfree method based on point interpolation technique and polynomial basic function, proposed by Liu and abbreviated as PIM [4], is implemented and developed to approximate the displacement field of the beam. The procedure for the approximation is as follows.

Scattering $n$ arbitrarily distributed nodes at the mid-plane and along the $x$-axis (1D beam model), the displacement field at the mid-plane of the beam could be approximated through the discrete displacement values at the nodes using the polynomial basic function: 
Bending Analysis of Sandwich Beam with Functionally Graded Face Sheets Using ... M. D. Do et al.

$$
\begin{gathered}
u_{o}(x)=\sum_{i=1}^{n} p_{u i}(x) \cdot a_{u i}=\left\{p_{u}(x)\right\}^{T}\left\{a_{u}\right\}, \\
w_{o}(x)=\sum_{i=1}^{2 n} p_{w i}(x) \cdot a_{w i}=\left\{p_{w}(x)\right\}^{T}\left\{a_{w}\right\}, \\
\phi_{o s}(x)=\sum_{i=1}^{n} p_{\phi i}(x) \cdot a_{\phi i}=\left\{p_{\phi}(x)\right\}^{T}\left\{a_{\phi}\right\}, \\
w_{o z}(x)=\sum_{i=1}^{n} p_{w z i}(x) \cdot a_{w z i}=\left\{p_{w z}(x)\right\}^{T}\left\{a_{w z}\right\}
\end{gathered}
$$

Coefficients and polynomial basic functions:

$$
\begin{aligned}
& \left\{a_{u}\right\}=\left\{\begin{array}{llll}
a_{u 1} & a_{u 2} & \ldots & a_{u n}
\end{array}\right\}^{T} ; \\
& \left\{a_{w}\right\}=\left\{\begin{array}{lllll}
a_{w 1} & a_{w 2} & \ldots & a_{w 2 n}
\end{array}\right\}^{T} \text {; } \\
& \left\{a_{\phi}\right\}=\left\{\begin{array}{llll}
a_{\phi 1} & a_{\phi 2} & \ldots & a_{\phi n}
\end{array}\right\}^{T} \text {; } \\
& \left\{a_{w z}\right\}=\left\{\begin{array}{lllll}
a_{w z 1} & a_{w z 2} & \ldots & a_{w z n}
\end{array}\right\}^{T}
\end{aligned}
$$

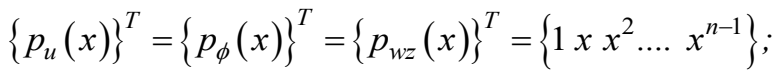

$$
\begin{aligned}
& \left\{p_{w}(x)\right\}^{T}=\left\{\begin{array}{llll}
1 & x & x^{2} & \ldots \\
x^{2 n-1}
\end{array}\right\}
\end{aligned}
$$

Note that $\left\{p_{w}(x)\right\}^{T}$ is chose to be $2 n$ terms because $w_{\mathrm{o}}$ is approximated to satisfy two degrees of freedom (deflection and derivatives of deflection) at each node.

The $(5 \times n)$ coefficients $\left\{a_{u}\right\},\left\{a_{w}\right\},\left\{a_{\phi}\right\},\left\{a_{w z}\right\}$ are determined by enforcing Eqs. (11)-(14) to be satisfied the displacement conditions at the nodes. Suppose that the nodal values of node $j$-th at $x=x_{j}$ are $\left\{q_{j}\right\}=\left\{u_{o j}, w_{o j}, \theta_{o j}, \phi_{o j}, w_{o z j}\right\}$, thus:

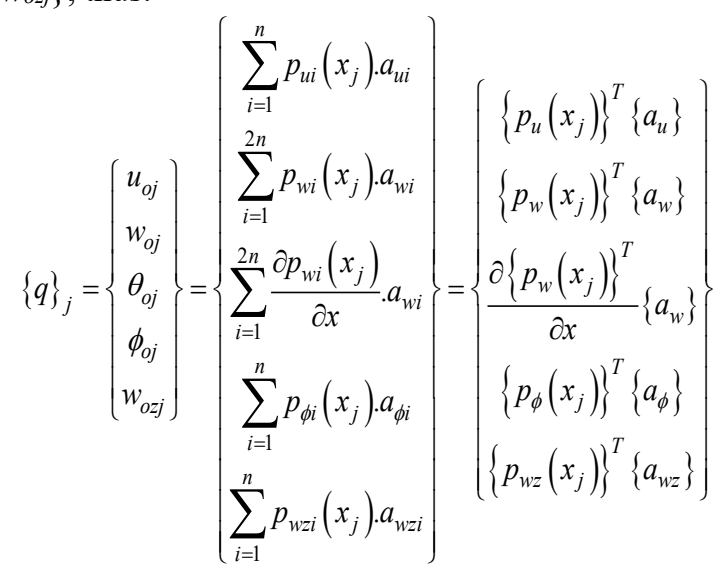

Rewriting Eq. (18) for all nodes $(j=1, \ldots, n)$ in the matrix form:

$$
\left.\{q\}=\left[P_{Q}\right]\left\{a_{u}\right\}\left\{a_{w}\right\}\left\{a_{\phi}\right\}\left\{a_{w z}\right\}\right\}^{T}=\left[P_{Q}\right]\{a\}
$$

Eq.(20) deduces the coefficients $\{a\}$ of polynomial approximation as

$$
\{a\}=\left[P_{Q}\right]^{-1}\{q\}
$$


Bending Analysis of Sandwich Beam with Functionally Graded Face Sheets Using ... M. D. Do et al.

By substituting the coefficients $\{a\}$ into Eqs. (11)-(14), the displacement field at the mid-plane is expressed in the nodal displacements $\{q\}$ :

$$
\left\{d_{o}\right\}=\left\{u_{o}(x) w_{o}(x) \phi_{o s}(x) w_{o z}(x)\right\}^{T}=p(x)^{T}\{a\}=p(x)^{T}\left[P_{Q}\right]^{-1}\{q\}
$$

Rearranging and replacing $u_{o}(x), w_{o}(x), \phi_{o s}(x), w_{o z}(x)$ from Eq. (21) into the energy expressions (Eqs. (9) and (10)), then applying the principle of virtual work, which has the mathematical form $\delta W^{\text {ext }}-\delta W^{\text {int }}=0$, we obtain the algebraic equations:

$$
[K]\{q\}=\{F\},
$$

where $[K]$ is stiffness matrix and $\{F\}$ is nodal load vector.

The nodal displacements, $\{q\}$, are determined by solving Eq. (22), then are used to calculate the strains and stresses.

\section{Numerical results and discussion}

FG sandwich beams subjected to a uniformly distributed load of magnitude $q_{o}$ is investigated for the case of simply supported edges. Mechanical properties of FGMs of the face sheets are ceramic (Alumina: $E_{c}=380 \mathrm{GPa}, v_{c}=0.3$ ) and metal (Aluminum: $E_{m}=70 \mathrm{GPa}, v_{m}=0.3$ ) - referred from [10] while homogeneous core is made of metal (Aluminum). Two cases of span-to-depth ratio $L / h=5$ (representing thick beams) and $L / h=20$ (representing thin beams) are considered. Results are reduced to be dimensionless as shown in following forms

$$
\bar{w}(x, z)=\frac{100 E_{m} b h^{3}}{q_{o} L^{4}} w(x, z) ; \bar{\sigma}_{x}(x, z)=\frac{b h}{q_{o} L} \sigma_{x}(x, z)
$$

\subsection{Convergence and validation study}

In Table 1, dimensionless values of axial stress at a specific position of 2-2-1 sandwich beam are obtained through the analysis by increasing number of uniformly scatted nodes. Also, for the purpose of comparison, the study is conducted with the consideration of FG sandwich homogeneous hard core

\begin{tabular}{|c|c|c|c|c|c|c|c|c|}
\hline \multirow{2}{*}{ Theories } & \multicolumn{7}{|c|}{ Number of nodes } & \multirow{2}{*}{$\begin{array}{c}\text { Vo et } \\
\text { al. } \\
{[10]}\end{array}$} \\
\hline & 2 & 3 & 4 & 5 & 6 & 7 & 8 & \\
\hline CBT & 1.04748 & 1.60027 & 1.57122 & 1.57122 & 1.57122 & 1.57122 & 1.57122 & - \\
\hline FBT & 1.04748 & 1.60027 & 1.57122 & 1.57122 & 1.57122 & 1.57122 & 1.57122 & 1.5712 \\
\hline TBT & 1.06361 & 1.61640 & 1.58739 & 1.58739 & 1.58732 & 1.58732 & 1.58743 & 1.5873 \\
\hline Quasi-3D & 1.06521 & 1.61456 & 1.58555 & 1.58423 & 1.58413 & 1.58563 & 1.58591 & 1.5849 \\
\hline
\end{tabular}
- only for this aim - which was studies by Vo et al. [10] using analytical solution and FEM. To achieve this goal, we just interchange the roles of $E_{m}$ and $E_{c}$ in the formulations in Section 2.1.

Table 1: Convergence study and comparison for the dimensionless axial stress $\bar{\sigma}_{x}\left(\frac{L}{2}, \frac{h}{2}\right)(L / h=5, k=2,2-2-1$ FG sandwich beam) 
Bending Analysis of Sandwich Beam with Functionally Graded Face Sheets Using ... M. D. Do et al.

The numerical results in Table 1 show that the convergence rate is amazingly fast. For CBT, FBT and TBT, the results have converged to 5 digits behind the decimal point for only the 4-node diagram required. It should be noted that quasi-3D beam theory gives a low rate convergence when compared to the other theories. Also, the results match well with those by Vo et al. [10] which confirms the model accuracy.

\subsection{Parametric studies}

In this subsection, the effects of slenderness ratios $(L / h)$, volume fraction indices, skin-core-skin thickness ratio and various beam theories on static behavior of the beams such as vertical displacement, axial stress are numerically investigated in detail. Note that only the soft-core sandwich beams are considered.

Table 2 presents the central dimensionless vertical displacement of the beams. As expected, the beams using CBT gives smaller deflections than those using the other theories because of ignoring shear deformation effect, and this effect is more pronounced in the thick beams $(L / h=5)$ than in the

\begin{tabular}{|c|c|c|c|c|c|c|c|c|c|}
\hline$L / h$ & Theories & $k$ & $1-0-1$ & $2-1-2$ & $2-1-1$ & $1-1-1$ & $2-2-1$ & $1-2-1$ & $1-8-1$ \\
\hline \multirow[t]{18}{*}{5} & & & & & & & & & 15.625 \\
\hline & CBT & 0 & 15.6250 & 15.6250 & 15.6250 & 15.6250 & 15.6250 & 15.6250 & 0 \\
\hline & & & & & & & & & 17.185 \\
\hline & FBT & 0 & 17.1850 & 17.1850 & 17.1850 & 17.1850 & 17.1850 & 17.1850 & 0 \\
\hline & & & & & & & & & 17.183 \\
\hline & TBT & 0 & 17.1830 & 17.1830 & 17.1830 & 17.1830 & 17.1830 & 17.1830 & 0 \\
\hline & & & & & & & & & 17.042 \\
\hline & Quasi-3D & 0 & 17.0425 & 17.0425 & 17.0425 & 17.0425 & 17.0425 & 17.0425 & 5 \\
\hline & CBT & 0.5 & 4.5901 & 4.9349 & 5.2824 & 5.2807 & 5.7451 & 5.9431 & 8.7560 \\
\hline & FBT & 0.5 & 5.2201 & 5.6502 & 6.0228 & 6.0670 & 6.5724 & 6.8407 & 9.9604 \\
\hline & & & & & & & & & 10.134 \\
\hline & TBT & 0.5 & 5.4018 & 5.9150 & 6.2562 & 6.3802 & 6.8575 & 7.1775 & 3 \\
\hline & & & & & & & & & 10.066 \\
\hline & Quasi-3D & 0.5 & 5.3634 & 5.8743 & 6.2048 & 6.3372 & 6.8017 & 7.1302 & 0 \\
\hline & CBT & 5 & 2.9208 & 3.0043 & 3.2264 & 3.1354 & 3.4537 & 3.4651 & 5.4693 \\
\hline & FBT & 5 & 3.2534 & 3.3990 & 3.6404 & 3.5863 & 3.9390 & 4.0134 & 6.3669 \\
\hline & TBT & 5 & 3.2952 & 3.5276 & 3.7483 & 3.8055 & 4.1445 & 4.3760 & 6.7608 \\
\hline & Quasi-3D & 5 & 3.2691 & 3.5012 & 3.7161 & 3.7784 & 4.1083 & 4.3471 & 6.7196 \\
\hline \multirow[t]{7}{*}{20} & & & & & & & & & 15.625 \\
\hline & CBT & 0 & 15.6250 & 15.6250 & 15.6250 & 15.6250 & 15.6250 & 15.6250 & 0 \\
\hline & & & & & & & & & 15.722 \\
\hline & FBT & 0 & 15.7225 & 15.7225 & 15.7225 & 15.7225 & 15.7225 & 15.7225 & 5 \\
\hline & & & & & & & & & 15.722 \\
\hline & TBT & 0 & 15.7225 & 15.7225 & 15.7225 & 15.7225 & 15.7225 & 15.7225 & 5 \\
\hline & Quasi-3D & 0 & 15.7140 & 15.7140 & 15.7140 & 15.7140 & 15.7140 & 15.7140 & 15.714 \\
\hline
\end{tabular}


Bending Analysis of Sandwich Beam with Functionally Graded Face Sheets Using ... M. D. Do et al.

\begin{tabular}{lllllllll}
\hline & & & & & & & & 0 \\
CBT & 0.5 & 4.5901 & 4.9349 & 5.2824 & 5.2807 & 5.7451 & 5.9431 & 8.7560 \\
FBT & 0.5 & 4.6295 & 4.9796 & 5.3287 & 5.3299 & 5.7968 & 5.9992 & 8.8313 \\
TBT & 0.5 & 4.6409 & 4.9963 & 5.3434 & 5.3496 & 5.8148 & 6.0204 & 8.8423 \\
Quasi-3D & 0.5 & 4.6386 & 4.9939 & 5.3316 & 5.3470 & 5.8015 & 6.0176 & 8.8382 \\
\hline CBT & 5 & 2.9208 & 3.0043 & 3.2264 & 3.1354 & 3.4537 & 3.4651 & 5.4693 \\
FBT & 5 & 2.9416 & 3.0290 & 3.2522 & 3.1636 & 3.4840 & 3.4994 & 5.5254 \\
TBT & 5 & 2.9443 & 3.0371 & 3.2590 & 3.1774 & 3.4970 & 3.5222 & 5.5502 \\
Quasi-3D & 5 & 2.9427 & 3.0355 & 3.2523 & 3.1758 & 3.4872 & 3.5204 & 5.5478 \\
\hline
\end{tabular}

Table 2: Dimensionless vertical displacements $\bar{w}\left(\frac{L}{2}, 0\right)$ of FG sandwich beams

thin ones $(L / h=20)$. For the case of homogeneous beam $(k=0)$, FBT and TBT give almost the same displacement values. However, $k \neq 0$, TBT gives slightly higher values than both FBT and quasi-3D theory. It is noted that the vertical displacement of beams increases as the layer thickness ratio increases from $1-0-1$ to $2-1-2$ to $2-1-1$ to $1-1-1$ to $2-2-1$ to $1-2-1$ to $1-8-1$ due to the increase in the soft-core thickness ratio. However, by comparison between the 2-1-1 and the 1-1-1 beam for some cases of $k$ and beam theories, the 2-1-1 beam gives a bit more deflection values than the 1-1-1 one does which is highlighted in red. This trend greatly affects the thin beam $(L / h=20)$ rather than the thick one $(L / h=5)$.

Dimensionless axial stress values at the top fiber of the central cross-section of the beams are listed in Table 3. It is worth noting that FBT and CBT give identical stress values and smaller in compared to TBT or to quasi-3D theory. In general, quasi-3D gives slightly higher axial stress values than those of TBT.

\begin{tabular}{|c|c|c|c|c|c|c|c|c|c|}
\hline$L / h$ & Theories & $k$ & $1-0-1$ & $2-1-2$ & $2-1-1$ & $1-1-1$ & $2-2-1$ & $1-2-1$ & $1-8-1$ \\
\hline \multirow[t]{12}{*}{5} & CBT & 0 & 3.7500 & 3.7500 & 3.7500 & 3.7500 & 3.7500 & 3.7500 & 3.7500 \\
\hline & FBT & 0 & 3.7500 & 3.7500 & 3.7500 & 3.7500 & 3.7500 & 3.7500 & 3.7500 \\
\hline & TBT & 0 & 3.8017 & 3.8017 & 3.8017 & 3.8017 & 3.8017 & 3.8017 & 3.8017 \\
\hline & Quasi-3D & 0 & 3.8011 & 3.8011 & 3.8011 & 3.8011 & 3.8011 & 3.8011 & 3.8011 \\
\hline & CBT & 0.5 & 5.9802 & 6.4295 & 7.5452 & 6.8800 & 8.2351 & 7.7431 & 11.4078 \\
\hline & FBT & 0.5 & 5.9802 & 6.4295 & 7.5452 & 6.8800 & 8.2351 & 7.7431 & 11.4078 \\
\hline & TBT & 0.5 & 6.1047 & 6.5744 & 7.6740 & 7.0370 & 8.3788 & 7.9099 & 11.5851 \\
\hline & Quasi-3D & 0.5 & 6.1091 & 6.5806 & 7.6792 & 7.0441 & 8.3850 & 7.9175 & 11.5904 \\
\hline & $\mathrm{CBT}$ & 5 & 3.8054 & 3.9142 & 4.5711 & 4.0850 & 5.0016 & 4.5145 & 7.1258 \\
\hline & FBT & 5 & 3.8054 & 3.9142 & 4.5711 & 4.0850 & 5.0016 & 4.5145 & 7.1258 \\
\hline & TBT & 5 & 3.8718 & 4.0043 & 4.6464 & 4.1955 & 5.0941 & 4.6515 & 7.2739 \\
\hline & Quasi-3D & 5 & 3.8726 & 4.0073 & 4.6488 & 4.2005 & 5.0983 & 4.6591 & 7.2817 \\
\hline \multirow[t]{4}{*}{20} & CBT & 0 & 15.0000 & 15.0000 & 15.0000 & 15.0000 & 15.0000 & 15.0000 & 15.0000 \\
\hline & FBT & 0 & 15.0000 & 15.0000 & 15.0000 & 15.0000 & 15.0000 & 15.0000 & 15.0000 \\
\hline & TBT & 0 & 15.0130 & 15.0130 & 15.0130 & 15.0130 & 15.0130 & 15.0130 & 15.0130 \\
\hline & Quasi-3D & 0 & 15.0127 & 15.0127 & 15.0127 & 15.0127 & 15.0127 & 15.0127 & 15.0127 \\
\hline
\end{tabular}


Bending Analysis of Sandwich Beam with Functionally Graded Face Sheets Using ... M. D. Do et al.

\begin{tabular}{lllllllll} 
CBT & 0.5 & 23.9209 & 25.7180 & 30.1808 & 27.5201 & 32.9405 & 30.9722 & 45.6313 \\
FBT & 0.5 & 23.9209 & 25.7180 & 30.1808 & 27.5201 & 32.9405 & 30.9722 & 45.6313 \\
TBT & 0.5 & 23.9522 & 25.7546 & 30.2132 & 27.5596 & 32.9767 & 31.0143 & 45.6760 \\
Quasi-3D & 0.5 & 23.9532 & 25.7560 & 30.2144 & 27.5613 & 32.9781 & 31.0161 & 45.6770 \\
\hdashline CBT & 5 & 15.2217 & 15.6569 & 18.2844 & 16.3401 & 20.0062 & 18.0581 & 28.5031 \\
FBT & 5 & 15.2217 & 15.6569 & 18.2844 & 16.3401 & 20.0062 & 18.0581 & 28.5031 \\
TBT & 5 & 15.2385 & 15.6796 & 18.3033 & 16.3679 & 20.0296 & 18.0927 & 28.5404 \\
Quasi-3D & 5 & 15.2385 & 15.6802 & 18.3038 & 16.3691 & 20.0305 & 18.0945 & 28.5423 \\
\hline
\end{tabular}

Table 3: Dimensionless axial stress $\bar{\sigma}_{x}\left(\frac{L}{2}, \frac{h}{2}\right)$ of FG sandwich beams

The influence of various beam theories and slenderness ratios on the central dimensionless deflection of the 1-2-1 beam is illustrated in Figure 2. It is found that the deflection value decreases with increasing $L / h$ ratio for all beam theories, except for CBT which gives constant value. For the thick beam with small $L / h$, the deviation of results calculated by the beam theories is significant, but negligible for the thin one (high $L / h$ ratio).

Effect of power-lax index $(k)$ on the central dimensionless deflection of the 1-2-1 beam is shown in Figure 3. When $k=0$, the sandwich beams become the homogeneous one of full metal, and the greatest deflection values are given. As $k$ increases gradually, the deflection values decrease because the ceramic component density, which has higher modulus, decreases. It could be found that the most sensitive effect of this index belongs to the range [0-2].

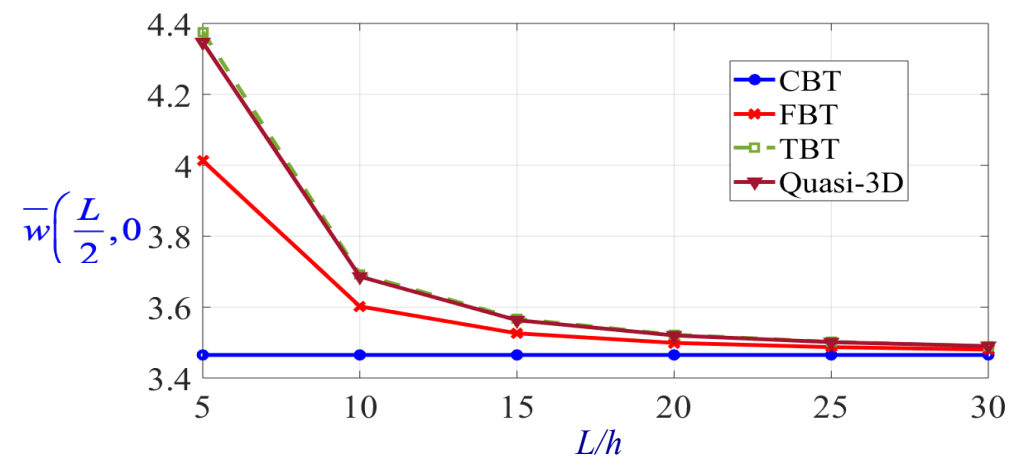

Figure 2: Variation of dimensionless deflections $\quad \bar{w}\left(\frac{L}{2}, 0\right)$ with respect to $L / h$ ratio $(1-2-1, k=5)$

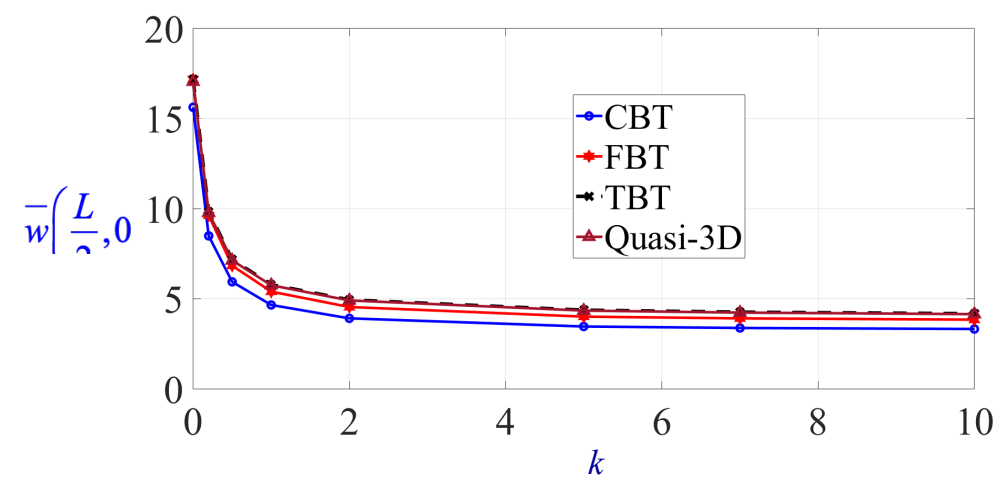

Figure 3: Variation of dimensionless deflections $\quad \bar{w}\left(\frac{L}{-}, 0\right)$ with respect to the power-law index $k\left(1-2-1, L / h_{\overline{14}}\right.$ ) 
Bending Analysis of Sandwich Beam with Functionally Graded Face Sheets Using ... M. D. Do et al.

\section{Conclusions}

A meshfree method based on point interpolation technique using polynomial basic function (PIM) has been well implemented and developed for static analysis behavior of sandwich beams with functionally graded face sheets. Various beam theories are taken into account in order to consider shear deform effects as well as compare results obtained among them. Four-unknown governing equation is derived from the principle of virtual work. The meshfree method using polynomial basic function along with the global approximation of the displacement field in the report is simple, fast convergence, efficient and accurate. Numerical examples are carried out to examine detailed effects of the span-to-height ratio $(L / h)$, the layer thicknesses ratio, the power-lax index $(k)$, the different beam theories on axial stress and vertical displacement. These effects are highly significant and should be considered in analysis and design of FG sandwich beams. The numerical results presented in tabular form is also a good source reference for future researches in order to compare results.

Acknowledgements. This work was supported by the University of Danang, University of Science and Technology, code number of Project: T2019-02-14.

\section{References}

[1] M.K.S.Sai, "Review of Composite Materials and Applications," vol. 6, no. 3, pp. 129-135, 2016.

[2] A.S.Sayyadandy, M.Ghugal, "Modeling and analysis of functionally graded sandwich beams: A review," Mechanics of Advanced Materials and Structure, vol. 26, no. 21, pp. 1776-1795, 2018.

[3] H.-S. Shen, Functionally graded materials: nonlinear analysis of plates and shells, Taylor \& Francis Group, 2009.

[4] G.R.Liu, Meshfree Methods: Moving Beyond the Finite Element Method, Boca Raton: CRC Press, 2002.

[5] A. Karamanl, "Bending behaviour of two directional functionally graded sandwich beams by using a quasi-3D shear deformation theory," Composite Structures, vol. 174, pp. 70-86, 2017.

[6] M.C. Amirani, S.M.R. Khalili, and N. Nemati, "Free vibration analysis of sandwich beam with FG core using the element free Galerkin method," Composite Structures, vol. 90, no. 3, pp. 373379, 2009.

[7] T.Q. Bui, A. Khosravifard, C. Zhang, M.R. Hematiyan, and M.V. Golub, "Dynamic analysis of sandwich beams with functionally graded core using a truly meshfree radial point interpolation method," Engineering Structures, vol. 47, pp. 90-104, 2013.

[8] Y. Yang, C.C. Lam, K.P. Kou, and V.P. Iu, "Free vibration analysis of the functionally graded sandwich beams by a meshfree boundary domain integral equation method," Composite Structures, vol. 117, pp. 32-39, 2014.

[9] A. I Osofero, T.P Vo, T-K Nguyen, J. Lee, "Analytical solution for vibration and buckling of functionally graded sandwich beams using various quasi-3D theories," Journal of Sandwich Structures and Materials, vol. 18, no. 1, pp. 3-29, 2015.

[10] T.P. Vo, H-T. Thai, T.-K. Nguyen, F. Inam, J. Lee, "Static behaviour of functionally graded sandwich beams using a quasi-3D theory," Composites Part B, vol. 68, pp. 59-74, 2015.

[11] T.P. Vo, H-T. Thai, T.-K. Nguyen, F. Inam, J. Lee, "A quasi-3D theory for vibration and buckling of functionally graded sandwich beams," Composite Structures, vol. 119, pp. 1-12, 
Bending Analysis of Sandwich Beam with Functionally Graded Face Sheets Using ... M. D. Do et al.

2015.

[12] T-K. Nguyen, T.P. Vo, B-D. Nguyen, J. Lee, "An analytical solution for buckling and vibration analysis of functionally graded sandwich beams using a quasi-3D shear deformation theory," Composite Structures, vol. 156, pp. 238-252, 2016. 